Militärs entwickelt hat, d. h. wie die beiden zentralen Aufgaben gelöst wurden, erstens dem größten Land Afrikas eine lebensfähige und gerechte föderative Verwaltungsstruktur zu geben, und zweitens, wie die diversen staatlichen Instanzen - Zentralregierung, die Regionalregierungen der 19 neu geschaffenen Bundesstaaten und die lokalen Councils - zur Entwicklung des Landes beigetragen haben und beitragen können.

Ein Schwerpunkt der Fallstudien konzentriert sich auf die Zusammenarbeit der Militärs mit den zivilen Instanzen, vor allem mit den Civil Servants und den Technokraten der halbstaatlichen Korporationen. Hierbei wird plausibel begründet, daß die Militärs nach der Machtübernahme - durch die erfolgreiche Beendigung des Bürgerkriegs zunächst gestärkt - keineswegs autonom regieren konnten, sondern in starkem Maße von zivilen Kräften abhängig waren. In einer höchst interessanten Fallstudie entwickelt Terisa Turner sogar die These, daß der Sturz von General Gowon im Juli 1975 nicht allein durch rivalisierende Militärs zu erklären ist, sondern durch unzufriedene Nigerianer aus dem kommerziellen Bereich (Kompradoren und Mittelsmänner zwischen ausländischen Firmen und der Staatsklasse), die sich bei der Verteilung des nationalen Olreichtums nach 1973 übergangen gefühlt haben.

Ein zweiter Schwerpunkt der Studien kreist um das Thema der gerechten Verteilung der Oleinnahmen zwischen Zentralregierung und Bundesstaaten. Es wird argumentiert, daß die seit Beginn der 70er Jahre angewachsene Bedeutung der Zentralregierung es den Offizieren ermöglicht hätte, die regionalen-ethnischen Tendenzen innerhalb des Landes (zwischen Haussas, Ibos und Yorubas) zu schwächen und dafür - gestützt auf die vergrößerte Finanzmacht von Lagos - die nationalen unitarischen Kräfte zu stärken. Durch die Militärs sei innerhalb einer neuen föderalistischen Struktur ein präsidentielles System geschaffen worden, das dem alten Westminister-Modell einer parlamentarischen Regierung überlegen sei.

Es ist ein gut recherchiertes, interessantes Buch, in dem unterschiedliche wissenschaftliche Ansätze und Meinungen vereint sind und das gerade deshalb Politologen, Soziologen und Wirtschafts- und Verwaltungswissenschaftlern als anregende Nigeria-Lektüre empfohlen werden kann.

Rainer Tetzlaff

\title{
Self-Housing Groups in Squatter Settlements of Zambia - Legal Aspects
}

hrsg. von der Carl-Duisberg-Gesellschaft e. V. (Köln) im Namen des Bundesministeriums für wirtschaftliche Zusammenarbeit in Kooperation mit der Deutschen Entwicklungshilfe für soziales Wohnungs- und Siedlungswesen e. V., Köln, 1983, 107 S.

Der vorliegende Band ist aus drei Seminaren entstanden, die die Carl Duisberg Gesellschaft e. V. (CDG) in Zusammenarbeit mit der Deutschen Entwicklungshilfe für soziales Wohnungs- und Siedlungswesen e. V. (DESWOS) und dem United Nations Centre for 
Human Settlements (UNCHS) in den Jahren 1980 bis 1982 in Marburg, Bundesrepublik Deutschland, in Nairobi, Kenia und in Lusaka, Zambia, durchgeführt hat. Ziel aller drei Seminare war "to study the use of the organizational form of integrated co-operatives for the improvement of housing and living conditions of low-income groups in developing countries and to elaborate adequate training programms for the promotion of participation and education in self-help groups" (S. 1), wobei sich die Untersuchung in ihrer dritten Phase in Lusaka wegen der in Zambia besonders selbsthilfefreundlichen Regierungs- und Verwaltungspolitik allein auf dieses Land bezog.

Die Erkenntnisse des Seminars in Lusaka sind im vorliegenden Band zusammengefaßt. Anstelle einer wissenschaftlich begründeten Ableitung der gewonnenen Ergebnisse gilt das Interesse der Herausgeber allein deren praktischer Umsetzung in der Arbeit mit Selbsthilfegruppen in den städtischen Siedlungsgebieten sogenannter "Squatter dwellers" (illegaler Siedler). Es werden deshalb auch keine unterschiedlichen wissenschaftlichen Standpunkte ausgebreitet, sondern allein die vor Ort relevanten Probleme der Einräumung eines Wohn- und Besitzrechtes der Siedler im Rahmen einer von den Behörden zu erteilenden, vererblichen Erlaubnis, die Zambia bereits in seinem "Housing (Statutory and Improvement Areas) Act" verwirklicht hat (vgl. S. 28), sowie Möglichkeiten der Organisation von Selbsthilfegruppen als eingetragener oder nicht eingetragener Verein bzw. als Genossenschaft (S. 33 ff.) erörtert. Dabei kommen auch die Möglichkeiten unterschiedlicher Beitragsleistungen durch die Mitglieder in Form von Geldbeiträgen bei Mitgliedern mit ständiger Arbeit und in Form von Arbeitsleistungen von einem bestimmten bei arbeitslosen oder nicht ständig beschäftigten Mitgliedern zur Sprache (S. 83 f.). Hinweise gelten auch der Finanzbeschaffung am Kapitalmarkt (S. 86 f.) sowie der praktischen und gleichberechtigten Ausführung der Tätigkeiten der Selbsthilfeorganisation (S. 89 ff.).

Die exemplarische Behandlung der Möglichkeiten zur Verbesserung der Wohn- und Lebenssituation von "squatter dwellers" durch Selbsthilfeorganisationen in Zambia sowie der an der Abfolge der Problemstellungen der Siedler und Selbsthilfegruppen orientierte Aufbau des Bandes und seine leichte Verständlichkeit, die nicht zuletzt durch die dem Text jeweils zugeordneten Abbildungen und Gesetzesauszügen bedingt ist, ermöglichen einerseits einen Einsatz des Buches als Handbuch für Entwicklungspromotoren vor Ort. Andererseits geben sie eindrücklich Aufschluß über die rechtlichen und organisatorischen Probleme dieser Siedlergruppen als Selbsthilfegruppen sowie deren Lösung in Zambia, die auch in anderen Ländern der Dritten Welt Nachahmung finden können.

Der Band ist für jeden, der sich mit Selbsthilfe-orientierter Entwicklungspolitik beschäftigt, von großem Wert. Auch wenn er nur den Ausschnitt Selbsthilfe im Siedlungsbereich umfaßt, kann er wertvolle Hinweise zur Selbsthilfe in anderen Bereichen liefern. ${ }^{1}$ Anderen mag er einen plastischen Eindruck in die Situation und akuten Probleme der Entwicklungsländer und Entwicklungspolitik geben.

Ulrich Werwigk

1 Vgl. Hans-H. Münkner, Die rechtliche Regelung der Vorgenossenschaft (VRU 1984, S. 261, Werwigk). 